\title{
Dexamethasone-induced production of reactive oxygen species promotes apoptosis via endoplasmic reticulum stress and autophagy in MC3T3-E1 cells
}

\author{
WANLIN LIU $^{1 *}$, ZHENQUN ZHAO $^{1 *}$, YUYAN NA $^{1}$, CHENYANG MENG $^{1}$, JIANZHONG WANG $^{2}$ and RUI BAI ${ }^{1}$ \\ Departments of ${ }^{1}$ Pediatric Orthopedics and ${ }^{2}$ Orthopedics and Trauma, \\ The Second Affiliated Hospital of Inner Mongolia Medical University, Hohhot, Inner Mongolia 010030, P.R. China
}

Received July 10, 2017; Accepted January 5, 2018

DOI: $10.3892 /$ ijmm.2018.3412

\begin{abstract}
Apoptosis of osteoblasts, triggered by prolonged or excessive use of glucocorticoids (GCs), has been identified as a dominant contributor to the development of osteoporosis and osteonecrosis. However, the molecular mechanisms underlying GC-induced apoptosis are multifaceted and remain to be fully elucidated. The present study aimed to explore the correlation between dexamethasone (DEX)-induced reactive oxygen species (ROS), autophagy and apoptosis in MC3T3-E1 osteoblast-like cells. Cell viability was assessed using a Cell Counting Kit- 8 assay, and flow cytometry was performed to assess cellular apoptosis, cell cycle and ROS production. Immunofluorescence and western blot analysis were respectively used to detect autophagic vacuoles and the expression of proteins, including cyclin D kinase (CDK)2, poly[ADP ribose] polymerase, caspase-3, activating transcription factor (ATF)4, CCAAT/enhancer-binding protein homologous protein (CHOP), Beclin1, microtubule-associated proteins 1A/1B light chain (LC)3B and P62. It was revealed that DEX not only reduced cell viability, but also promoted apoptosis via the activation of endoplasmic reticulum (ER) stress. In addition,
\end{abstract}

Correspondence to: Professor Rui Bai, Department of Pediatric Orthopedics, The Second Affiliated Hospital of Inner Mongolia Medical University, 1 Yingfang Road, Hohhot, Inner Mongolia 010030, P.R. China

E-mail: bairuiandaiqing@126.com

Professor Jianzhong Wang, Department of Orthopedics and Trauma, The Second Affiliated Hospital of Inner Mongolia Medical University, 1 Yingfang Road, Hohhot, Inner Mongolia 010030, P.R. China

E-mail:wjzwan@126.com

${ }^{*}$ Co-first authorship

Abbreviations: DEX, dexamethasone; ROS, reactive oxygen species; ER, endoplasmic reticulum; NAC, N-acetylcysteine

Key words: dexamethasone, reactive oxygen species, autophagy, endoplasmic reticulum stress, MC3T3-E1 cells
DEX induced cell cycle arrest at G0/G1 phase via inhibition of the expression of CDK2, and the production of ROS was activated. Of note, the DEX-mediated changes in viability and apoptosis were attenuated in MC3T3-E1 cells after treatment with 3-methyladenine, which is an autophagy inhibitor. Treatment with the antioxidant $\mathrm{N}$-acetylcysteine abolished the effect of DEX on the proliferation, apoptosis, ER stress and autophagy of MC3T3-E1 cells. In conclusion, the present results indicated that DEX promoted the production of ROS, which enhanced apoptosis through activation of autophagy and ER stress in MC3T3-E1 cells.

\section{Introduction}

Glucocorticoids (GCs) are naturally produced steroid hormones or synthetic compounds that are frequently used as anti-inflammatory and immune-suppressive drugs to treat a variety of diseases. However, there is evidence that prolonged or excessive use of GCs is one of the leading causes of osteoporosis and osteonecrosis (1-3). It has been demonstrated that GCs induce fractures in $30-50 \%$, and osteonecrosis in $9-40 \%$ of patients receiving long-term therapy (4). GCs not only directly suppress the osteogenic differentiation of osteoblasts, but they also induce osteoblast and osteocyte apoptosis $(5,6)$. Furthermore, apoptosis of osteocytes is the dominant mechanism of GC-induced osteoporosis (7).

Reactive oxygen species (ROS) are the derivatives of biological aerobic metabolism, comprising hydrogen peroxide, hydroxyl radicals and superoxide. ROS destroy and oxidize proteins, lipids and DNA, leading to altered cell function. It is thought that ROS are generated under various physiological conditions, but also contribute to the pathogenesis of bone loss, including that in osteoporosis (8-11). Autophagy is a major intracellular degradation process, by which cytoplasmic proteins and organelles are sequestered in double-membrane vesicles and degraded upon fusion with lysosomes (12). Autophagy principally acts as a form of cytoprotection via maintaining the homeostasis of intracellular nutrients and energy. Autophagy is associated with a large number of physiological processes, as well as pathophysiological conditions and diseases, including neurodegeneration and microbial infection (13). 
Dexamethasone (DEX), a synthetic GC hormone, has been identified to inhibit the synthesis of fibronectin and collagen, and to activate collagenase synthesis. Evidence has indicated that DEX promotes osteoblast apoptosis by activating the expression of caspase family proteins (14). However, the involvement of ROS and autophagy in the apoptosis of osteoblasts caused by GCs and the exact underlying mechanisms have remained to be fully elucidated. Therefore, the purpose of the present study was to measure the pharmacological effect of DEX on ROS and autophagy in the osteoblast-like MC3T3-E1 cell line. Furthermore, it was investigated whether DEX induced apoptosis of MC3T3-E1 cells via triggering ROS production and autophagy.

\section{Materials and methods}

Cell culture. MC3T3-E1 cells (American Type Culture Collection, Manassas, VA, USA) were grown in Dulbecco's modified Eagle's medium (high glucose; Gibco; Thermo Fisher Scientific, Inc., Waltham, MA, USA) supplemented with $10 \%$ fetal bovine serum (Gibco; Thermo Fisher Scientific, Inc.) and penicillin-streptomycin solution $(100 \mathrm{U} / \mathrm{ml}$ penicillin; $100 \mu \mathrm{g} / \mathrm{ml}$ streptomycin; Gibco; Thermo Fisher Scientific, Inc.), in a humidified atmosphere with $5 \% \mathrm{CO}_{2}$ at $37^{\circ} \mathrm{C}$.

Cell viability assay. Cell viability assessment was performed using a Cell Counting Kit-8 (CCK-8; Dojindo Molecular Technologies, Inc., Kumamoto, Japan). MC3T3-E1 cells (1x10\% /well) were seeded in 96-well plates, incubated overnight and then treated with DEX at various concentrations 0.1 , 1 and $10 \mu \mathrm{M}$ for $24 \mathrm{~h}$. The absorbance values at $450 \mathrm{~nm}$ were measured using a microplate reader (Bio-Rad Laboratories, Inc., Hercules, CA, USA). Furthermore, in order to assess the connection of DEX-induced autophagy with apoptosis, 3-methyladenine (3-MA; Sigma-Aldrich; Merck KGaA, Darmstadt, Germany) was used as a common anti-autophagy factor. Cells were divided into four groups, including normal control, $1 \mu \mathrm{M}$ DEX, $5 \mathrm{mM}$ 3-MA (cells cultured with $5 \mathrm{mM}$ 3-MA for $24 \mathrm{~h}$ ) and $5 \mathrm{mM} 3-\mathrm{MA}+1 \mu \mathrm{M}$ DEX group (cells cultured with $5 \mathrm{mM} 3-\mathrm{MA}+1 \mu \mathrm{M}$ DEX for $24 \mathrm{~h}$ ). In addition, in order to investigate the association between ROS induced by DEX and cell apoptosis, $10 \mathrm{mM}$ N-acetylcysteine (NAC; Beyotime Institute of Biotechnology, Haimen, China) was added to MC3T3-E1 cells treated with DEX. NAC is a commonly used antioxidant. Cells were divided into normal group, $1 \mu \mathrm{M}$ DEX group, $10 \mathrm{mM}$ NAC group (cells cultured with $10 \mathrm{mM}$ NAC for $24 \mathrm{~h}$ ) and $10 \mathrm{mM} \mathrm{NAC}+1 \mu \mathrm{M}$ DEX group (cells cultured with $10 \mathrm{mM} \mathrm{NAC}+1 \mu \mathrm{M}$ DEX for $24 \mathrm{~h}$ ).

Analysis of cellular apoptosis. An Annexin V-fluorescein isothiocyanate (FITC) apoptosis Detection kit (KeyGen Biotech Co., Ltd., Nanjing, Jiangsu, China) was used to determine the level of cellular apoptosis. MC3T3-E1 cells (4x10\% $/$ well) were cultured in 6-well plates overnight and then treated as described above. In brief, cells were suspended in $500 \mu 11 \mathrm{X}$ binding buffer and incubated in the dark with $5 \mu \mathrm{l}$ Annexin V-FITC and $5 \mu \mathrm{l}$ propidium iodide (PI) for $10 \mathrm{~min}$ at room temperature. A flow cytometer (FACSCalibur; BD Biosciences, Franklin Lakes, NJ, USA) was used to analyze cellular apoptosis.
Analysis of the cell cycle. A Cell Cycle Detection kit (KeyGen Biotech Co., Ltd.) was used to assess the cell cycle. Following treatment with DEX, the MC3T3-E1 cells were trypsinized, collected and washed with PBS. Subsequently, 70\% cold ethanol was added to fix the cells for $2 \mathrm{~h}$ at room temperature or overnight at $4^{\circ} \mathrm{C}$, and $\mathrm{PBS}$ was used to wash away the fixing solution. Cells were incubated with $0.4 \mathrm{ml}$ PI containing $0.1 \mathrm{ml}$ RNase A at $37^{\circ} \mathrm{C}$ for $30 \mathrm{~min}$. Finally, cell cycle distribution was analyzed by measuring the DNA content using a flow cytometer.

Monodansylcadaverine (MDC) staining. Autophagy is characterized by the formation of acidic vesicles, as described previously, and MDC is often used as a selective fluorescent marker for autophagic vacuoles in vivo (15). MC3T3-E1 cells (4x10\% $/$ well) were seeded onto sterile coverslips placed in a 6-well plate and allowed to attach for $24 \mathrm{~h}$. As aforementioned, $0.05 \mathrm{mmol} / 1 \mathrm{MDC}$ in PBS was added to label the autophagic vacuoles for $10 \mathrm{~min}$ at $37^{\circ} \mathrm{C}$, and cells were washed three times with PBS. MC3T3-E1 cells were observed under a fluorescence microscope (Olympus BX53; Olympus Corp., Tokyo, Japan). Excitation wavelength was $335 \mathrm{~nm}$, and emission wavelength was $512 \mathrm{~nm}$. Average fluorescence intensity analysis was performed using Image-Pro Plus v6.0 software (Media Cybernetics Inc., Rockville, MD, USA).

ROS detection. A ROS Assay kit (KeyGen Biotech Co., Ltd.) was used for active ROS detection using the fluorescent probe 2',7'-dichlorofluorescin diacetate (DCFH-DA), which is a type of ROS-sensitive dye (16). The DCFH-DA reagent must be diluted to $10 \mu \mathrm{mol} / 1$ in serum-free medium prior to use. Following the experimental treatments, $10 \mu \mathrm{mol} / 1 \mathrm{DCFH}-\mathrm{DA}$ was added to cells with subsequent incubation for $30 \mathrm{~min}$ at $37^{\circ} \mathrm{C}$ in a humidified incubator, and cells were washed three times with serum-free medium and collected. A flow cytometer was used to quantify the relative fluorescence intensities.

Western blot analysis. Total protein was extracted from treated MC3T3-E1 cells with radioimmunoprecipitation assay lysis buffer (Tris $50 \mathrm{mM}, \mathrm{NaCl} 150 \mathrm{mM}$, Nonidet P-40 $1 \%$, sodium deoxycholate $0.5 \%$ and SDS $0.1 \%$; Beyotime Institute of Biotechnology, Haimen, China) containing $1 \%$ phenylmethylsulfonyl fluoride $(100 \mathrm{mM}$; Beyotime Institute of Biotechnology). The lysates were centrifuged at $12,000 \mathrm{xg}$ for $15 \mathrm{~min}$ at $4^{\circ} \mathrm{C}$. Supernatants were collected and the bicinchoninic acid protein assay kit (Beyotime Institute of Biotechnology) was used to detect the protein concentration. Equivalent amounts $(25 \mu \mathrm{g})$ of protein were separated by $12 \%$ SDS-PAGE and subsequently transferred onto a polyvinylidene difluoride membrane (Merck KGaA). Membranes were blocked with $5 \%$ non-fat milk in Tris-buffered saline with Tween-20 (0.05\%) at room temperature for $1 \mathrm{~h}$ and incubated overnight with primary antibodies. Following incubation with the corresponding secondary antibody, horseradish peroxidase-conjugated anti-rabbit immunoglobulin G (1:10,000 dilution; cat. no. 7074P2; Cell Signaling Technology, Inc., Danvers, MA, USA), at room temperature for $2 \mathrm{~h}$, membranes were washed three times with PBS. Finally, according to the manufacture's protocols, the blotting proteins were visualized with ECL plus reagent (Invitrogen; Thermo Fisher 
Scientific, Inc.). Antibodies used for the western blot analysis in the present study were as follows: Rabbit anti-activating transcription factor 4 (ATF4; 1:1,000 dilution; cat. no. 11815), rabbit anti-CCAAT/enhancer-binding protein homologous protein (CHOP; 1:1,000 dilution; cat. no. 2895), rabbit anti-poly[ADP ribose] polymerase (total PARP; 1:2,000 dilution; cat. no. 9542), rabbit anti-caspase-3 (total caspase-3; 600 dilution; cat. no. 96621) were obtained from Cell Signaling Technology, Inc. In addition, rabbit anti-Beclin1 (1:1,000 dilution, cat. no. ab62557), rabbit anti-LC3B (1:1,000 dilution, cat. no. ab51520), rabbit anti-P62 (1:1,000 dilution; cat. no. ab91526) and rabbit anti-cyclin-dependent kinase 2 (CDK2; 1:1,000 dilution; cat. no. ab32147) were obtained from Abcam (Cambridge, UK). Additionally, rabbit anti-GAPDH (1:1,000 dilution; cat. no. AB-P-R 001; Hangzhou Goodhere Biotechnology Co., Ltd., Hangzhou, China) was used as loading control. ImageJ v1.47 software (National Institutes of Health, Bethesda, MD, USA) was used to perform relative intensity of each protein band analysis.

Statistical analysis. All experiments were performed three times. Statistical analysis was performed with SPSS 19.0 statistical software (IBM Corp., Armonk, NY, USA). One-way analysis of variance followed by Tukey's post hoc test was used for multiple comparisons, and Student's t-test for comparisons between two groups. Values are expressed as the mean \pm standard deviation. $\mathrm{P}<0.05$ was considered to indicate a statistically significant difference.

\section{Results}

DEX inhibits viability and enhances apoptosis via endoplasmic reticulum (ER) stress in MC3T3-E1 cells. The effect of DEX on cell viability was evaluated with a CCK-8 assay. As illustrated in Fig. 1A, the amount of viable MC3T3-E1 cells was significantly inhibited by DEX in a dose-dependent manner (all $\mathrm{P}<0.01$ ), and at $10 \mu \mathrm{M} \mathrm{DEX}$, the cell viability reached $70 \%$ of that of the control group. In addition, the cellular apoptosis assay demonstrated that compared with that in the control group $(2.73 \%)$, the apoptotic rates in the DEX-treated groups were 7.9, 17.2 and $21.07 \%$ at DEX concentrations of $0.1,1$ and $10 \mu \mathrm{M}$, respectively $(\mathrm{P}<0.05, \mathrm{P}<0.01$ and $\mathrm{P}<0.01$, respectively; Fig. $1 \mathrm{~B}$ and $\mathrm{C}$ ).

Furthermore, western blotting was performed to evaluate the expression of PARP and caspase-3, which are early markers of apoptosis. As presented in Fig. 1D and E, DEX increased the activation of caspase-3 and PARP, which further confirmed that DEX promoted apoptosis of MC3T3-E1 cells. These results demonstrated that DEX not only reduces cell viability, but also promotes apoptosis. In addition, CHOP and ATF4 are key factors of ER stress. The expression of CHOP and ATF4 was gradually enhanced with increasing concentration of DEX (Fig. 1D and F). These results indicated that DEX may promote apoptosis by activating ER stress.

DEX causes G0/G1 arrest of MC3T3-E1 cells. The results of the cell cycle analysis are presented in Fig. 2A and B. DEX at concentrations of 1 and $10 \mu \mathrm{M}$ induced a significant increase in the number of cells in G0/G1 phase, but decreased the number of cells in S phase, indicating that DEX inhibited cell cycle progression (both $\mathrm{P}<0.05$ ). Subsequently, the expression of CDK2, which is essential for the G1/S transition, was detected. As illustrated in Fig. 2C and D, the expression of CDK2 was inhibited in MC3T3-E1 cells after treatment with DEX at concentrations of 1 and $10 \mu \mathrm{M}(\mathrm{P}<0.05, \mathrm{P}<0.01)$. These results suggested that DEX may induce G0/G1 arrest in MC3T3-E1 cells due to decreased CDK2 protein expression.

Effect of DEX on autophagy of MC3T3-E1 cells. To investigate the effect of DEX on autophagy in MC3T3-E1 cells, MDC staining and western blotting were performed. After treatment with DEX for $24 \mathrm{~h}$, fluorescence intensity was analyzed (Fig. 3A and B). The average fluorescence intensities in the $0.1,1$ and $10 \mu \mathrm{M}$ DEX-treated groups were increased to $0.18,0.22$ and 0.25 , respectively, which were significantly different from the average fluorescence intensity of the control group, $0.11(\mathrm{P}<0.05, \mathrm{P}<0.01$ and $\mathrm{P}<0.01$, respectively). Furthermore, in DEX $(1 \mu \mathrm{M})$ and $\operatorname{DEX}(10 \mu \mathrm{M})$ groups, the expression levels of autophagy markers, including LC3B-II, Beclin1 and P62, were gradually increased, but the levels of P62 were reduced, indicating the stimulatory role of DEX on autophagic flux $(\mathrm{P}<0.05, \mathrm{P}<0.01$; Fig. $3 \mathrm{C}$ and $\mathrm{D}$, respectively). These results indicated that DEX may promote the autophagy of MC3T3-E1 cells.

Association between autophagy and apoptosis in MC3T3-E1 cells. 3-MA inhibits autophagy by blocking autophagosome formation via the inhibition of class III phosphoinositide-3 kinase (17). To clarify the possible association between autophagy and apoptosis after treatment with DEX, the effect of 3-MA on viability and apoptosis was investigated. MC3T3-E1 cells were treated with DEX $(1 \mu \mathrm{M}), 3-\mathrm{MA}$ $(5 \mathrm{mM})$ or $3-\mathrm{MA}(5 \mathrm{mM})+\mathrm{DEX}(1 \mu \mathrm{M})$. As demonstrated in Fig. 4A, the viability of MC3T3-E1 cells decreased to $76.5 \%$ in the presence of DEX $(1 \mu \mathrm{M})$; however, after treatment with 3-MA $(5 \mathrm{mM})+\mathrm{DEX}(1 \mu \mathrm{M})$, the viability was significantly increased to $90.1 \%(\mathrm{P}<0.001)$. Furthermore, as presented in Fig. 4B and C, the apoptotic rate of MC3T3-E1 cells treated with DEX $(1 \mu \mathrm{M})$ was $24.3 \%$, while it significantly decreased to $18.56 \%$ in the $3-\mathrm{MA}(5 \mathrm{mM})+\operatorname{DEX}(1 \mu \mathrm{M})$ group $(\mathrm{P}<0.05)$. Inconsistencies in the apoptotic proportion in the $\operatorname{DEX}(1 \mu \mathrm{M})$ group between Figs. 1C and Fig. 4C may be due to technical issues, including different batches of kit. These results indicated that 3-MA reversed the DEX-induced inhibition of viability and promotion of apoptosis in MC3T3-E1 cells.

Furthermore, the expression levels of apoptosis- and autophagy-associated proteins were detected (Fig. 4D). The expression levels of the autophagy-associated proteins LC3B-II and Beclin1 were increased in the $\operatorname{DEX}(1 \mu \mathrm{M})$ group, which was inhibited by simultaneous treatment with 3-MA (5 mM; $\mathrm{P}<0.05$; Fig. 4E). In addition, it was demonstrated that the activation/expression of the apoptosis-associated proteins PARP and caspase-3 was weakened after treatment with 3-MA $(5 \mathrm{mM})+\mathrm{DEX}(1 \mu \mathrm{M})$, compared with that in the DEX $(1 \mu \mathrm{M})$ group $(\mathrm{P}<0.05$; Fig. 4F). These results suggested that DEX may promote MC3T3-E1 cell apoptosis by inducing autophagy.

DEX triggers ROS-dependent ER stress and autophagy to induce apoptosis. To examine how DEX affects the production of ROS in MC3T3-E1 cells, flow cytometry was performed to 
A

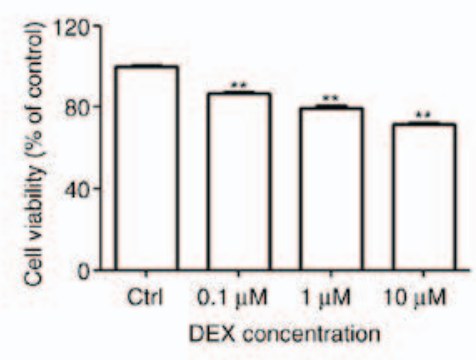

C

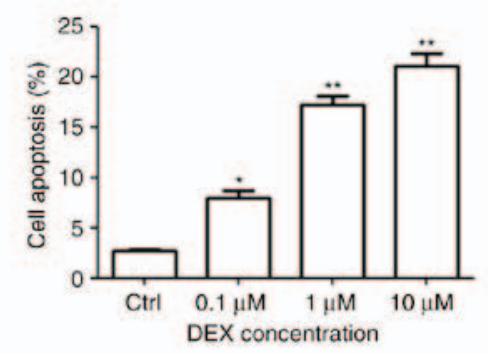

E

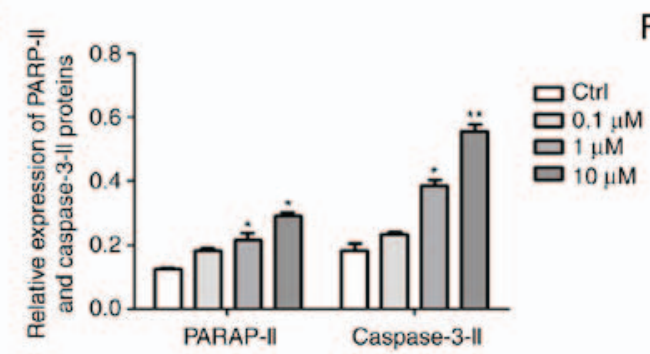

B

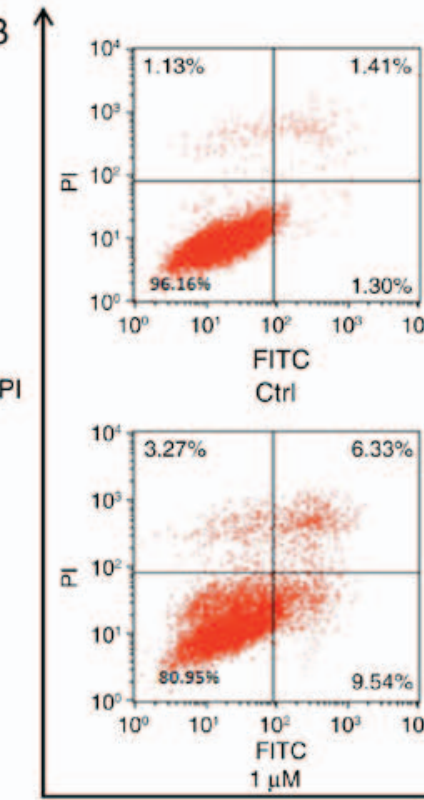

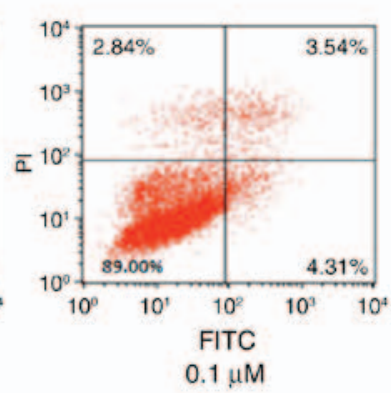

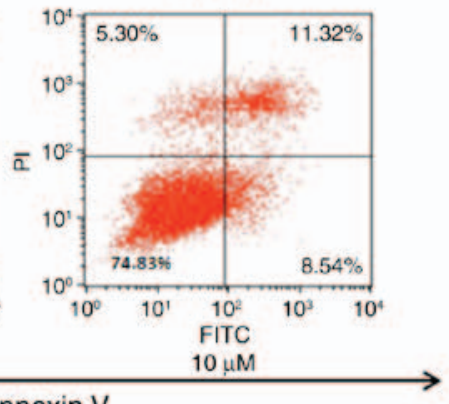

Annexin V

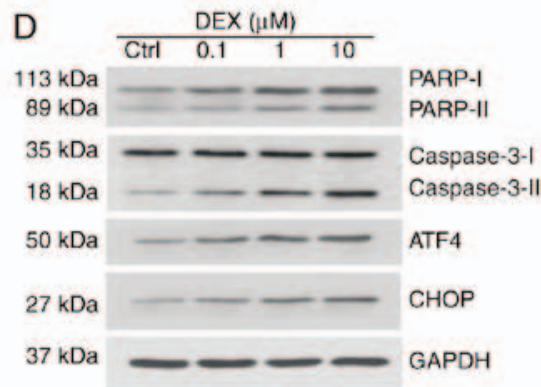

$\mathrm{F}$

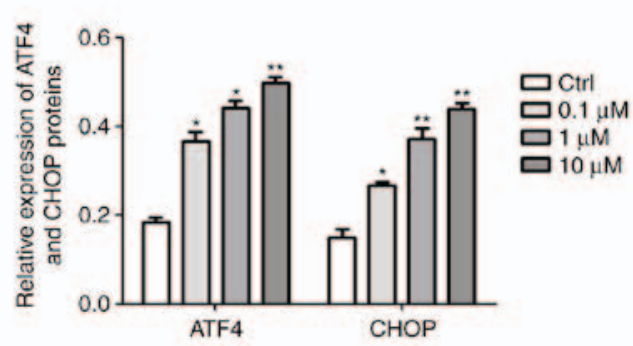

Figure 1. DEX induces ER stress and apoptosis in MC3T3-E1 cells. (A) The viability and (B and C) apoptosis of MC3T3-E1 cells were affected by DEX in a dose-dependent manner, as determined by a Cell Counting Kit-8 assay and Annexin V-FITC/PI staining, respectively. (D) Western blot analysis of ER stress-associated proteins (ATF4 and CHOP) and apoptosis-associated proteins (PARP and caspase-3). Values are expressed as the mean \pm standard deviation $(n=3)$. $\left(E\right.$ and F) Data from intensities analyses are presented as the relative expression of each protein to GAPDH. ${ }^{*} \mathrm{P}<0.05$ and ${ }^{* *} \mathrm{P}<0.01 \mathrm{vs}$. Ctrl. Ctrl, control; ATF, activating transcription factor; CHOP, CCAAT/enhancer-binding protein homologous protein; DEX, dexamethasone; ER, endoplasmic reticulum; PARP, poly[ADP ribose] polymerase; PI, propidium iodide; FITC, fluorescein isothiocyanate.

quantify the fluorescence intensity. In this experiment, NAC was used as an antioxidant. As illustrated in Fig. 5A and B, the mean ROS-associated fluorescence intensity in the DEX $(1 \mu \mathrm{M})$ group was increased compared with that in the control group, which was significantly inhibited by simultaneous treatment with NAC $(10 \mathrm{mM} ; \mathrm{P}<0.01)$. Furthermore, the cell viability in the NAC $(10 \mathrm{mM})+\mathrm{DEX}(1 \mu \mathrm{M})$ group was $89.3 \%$ of that in the control group, which was significantly higher than that in the DEX $(1 \mu \mathrm{M})$ group (77.8\% of control; $\mathrm{P}<0.01$; Fig. 5C). These results demonstrate that NAC inhibited the effect of DEX on MC3T3-E1 cell proliferation following co-treatment. Furthermore, cellular apoptosis was detected (Fig. 5D and E), revealing that the apoptotic rate of
MC3T3-E1 cells treated with NAC $(10 \mathrm{mM})+\mathrm{DEX}(1 \mu \mathrm{M})$ was $17.7 \%$, which was significantly decreased compared with that in the DEX $(1 \mu \mathrm{M})$ group $(25.97 \%$; $\mathrm{P}<0.01)$. These results indicated that activation of intracellular ROS was involved in the DEX-induced inhibition of viability and the promotion of apoptosis in MC3T3-E1 cells.

Subsequently, the expression of autophagy- and ER stress-associated proteins was quantified by western blot analysis (Fig. 5F). As illustrated in Fig. 5G, the protein levels of LC3B-II and Beclin1 were decreased, but P62 expression was increased in the NAC $(10 \mathrm{mM})+\operatorname{DEX}(1 \mu \mathrm{M})$ group when compared with that in the DEX $(1 \mu \mathrm{M})$ group $(\mathrm{P}<0.05)$. This result indicated that DEX activated autophagy via inducing 

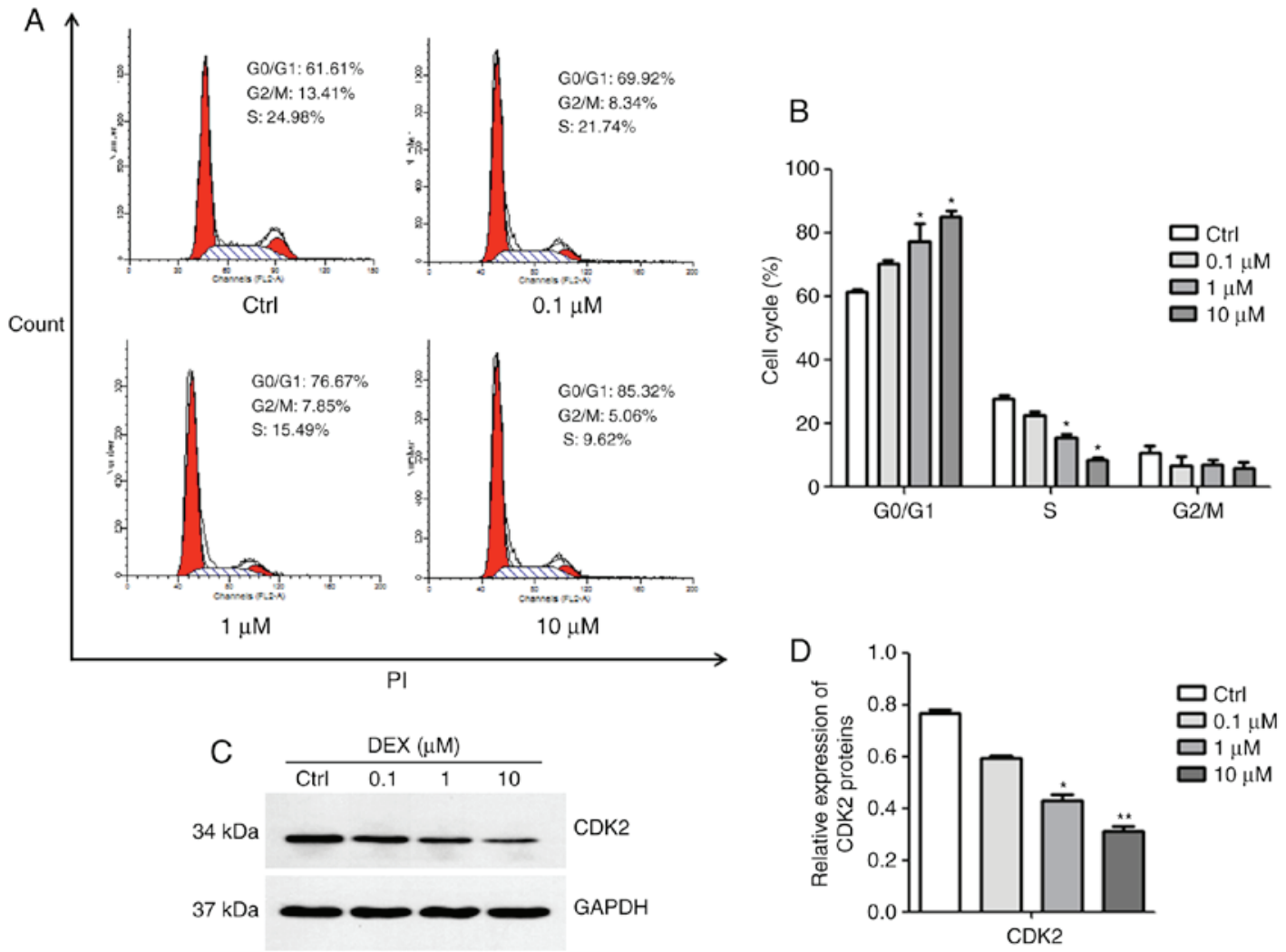

Figure 2. DEX induces G0/G1 phase arrest in MC3T3-E1 cells. (A) Cell cycle analysis using flow cytometry following PI staining and (B) quantification of the cell cycle populations demonstrated that the proportion of cells in G0/G1 phase increased in the DEX treatment group compared with that in the control. (C) The expression of CDK2 was assessed by western blot analysis. (D) A relative quantification assay was performed to assess the expression of CDK2 normalized to the GAPDH expression. Values are expressed as the mean \pm standard deviation $(\mathrm{n}=3)$. ${ }^{*} \mathrm{P}<0.05$ and ${ }^{* *} \mathrm{P}<0.01$ vs. Ctrl. Ctrl, control; CDK, cyclin D kinase; DEX, dexamethasone; PI, propidium iodide.

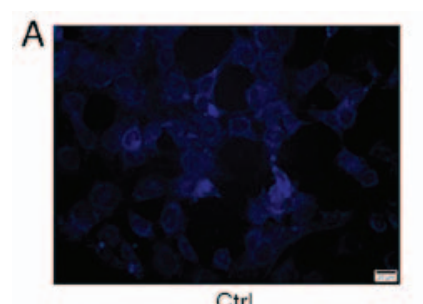

Ctrl

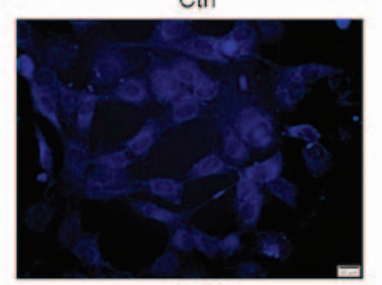

$1 \mu \mathrm{M}$

C

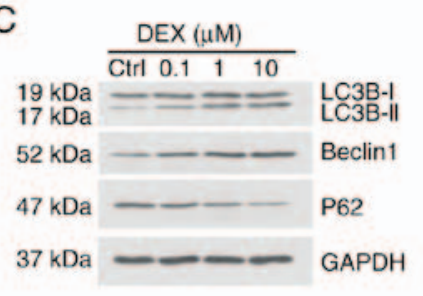

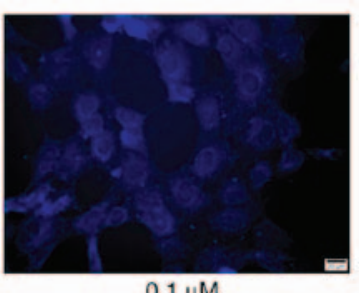

$0.1 \mu \mathrm{M}$
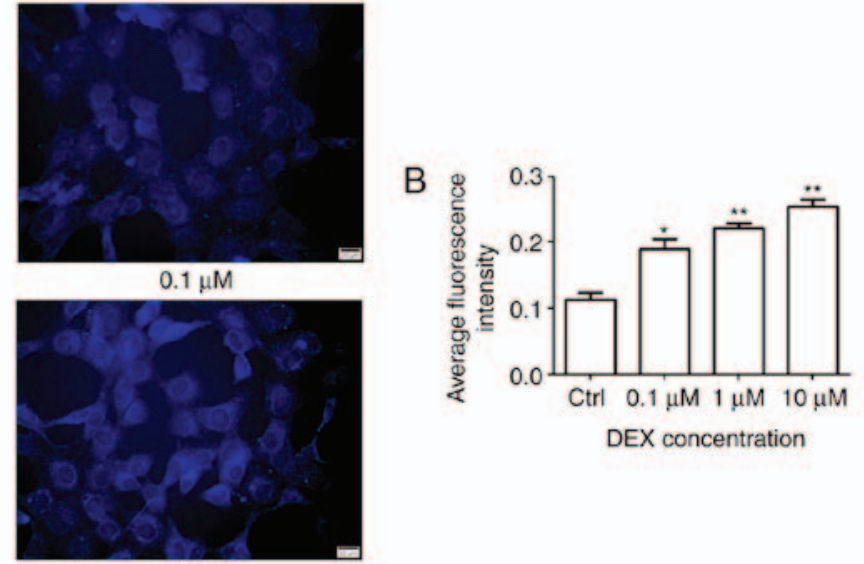

$10 \mu \mathrm{M}$

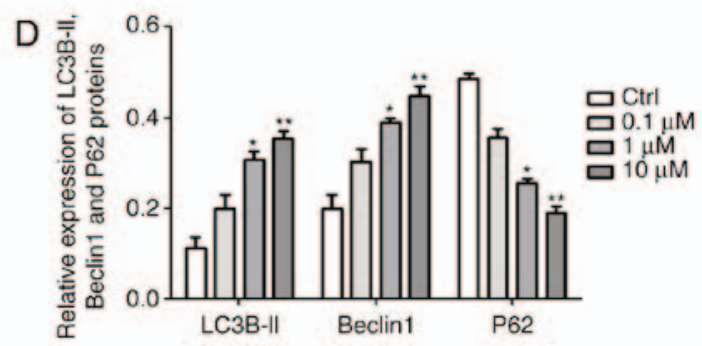

Figure 3. Fluorescence intensity of autophagic vacuoles in MC3T3-E1 cells under fluorescence microscopy. (A) The intensity of autophagic vacuoles was evaluated by MDC staining (magnification, $\mathrm{x} 400$; scale bar, $20 \mu \mathrm{m}$ ) and (B) their average fluorescence intensity was quantified. It was indicated that DEX increased the number of autophagic vacuoles. (C) Western blot analysis was performed to determine the protein expression levels of autophagy-associated proteins Beclin1, LC3B and P62. (D) The relative expression of LC3B-II, Beclin1 and P62 were calculated. Values are expressed as the mean \pm standard deviation $(n=3) .{ }^{~} \mathrm{P}<0.05$ and ${ }^{* *} \mathrm{P}<0.01$ vs. Ctrl. Ctrl, control; DEX, dexamethasone; LC3B, microtubule-associated proteins 1A/1B light chain. 

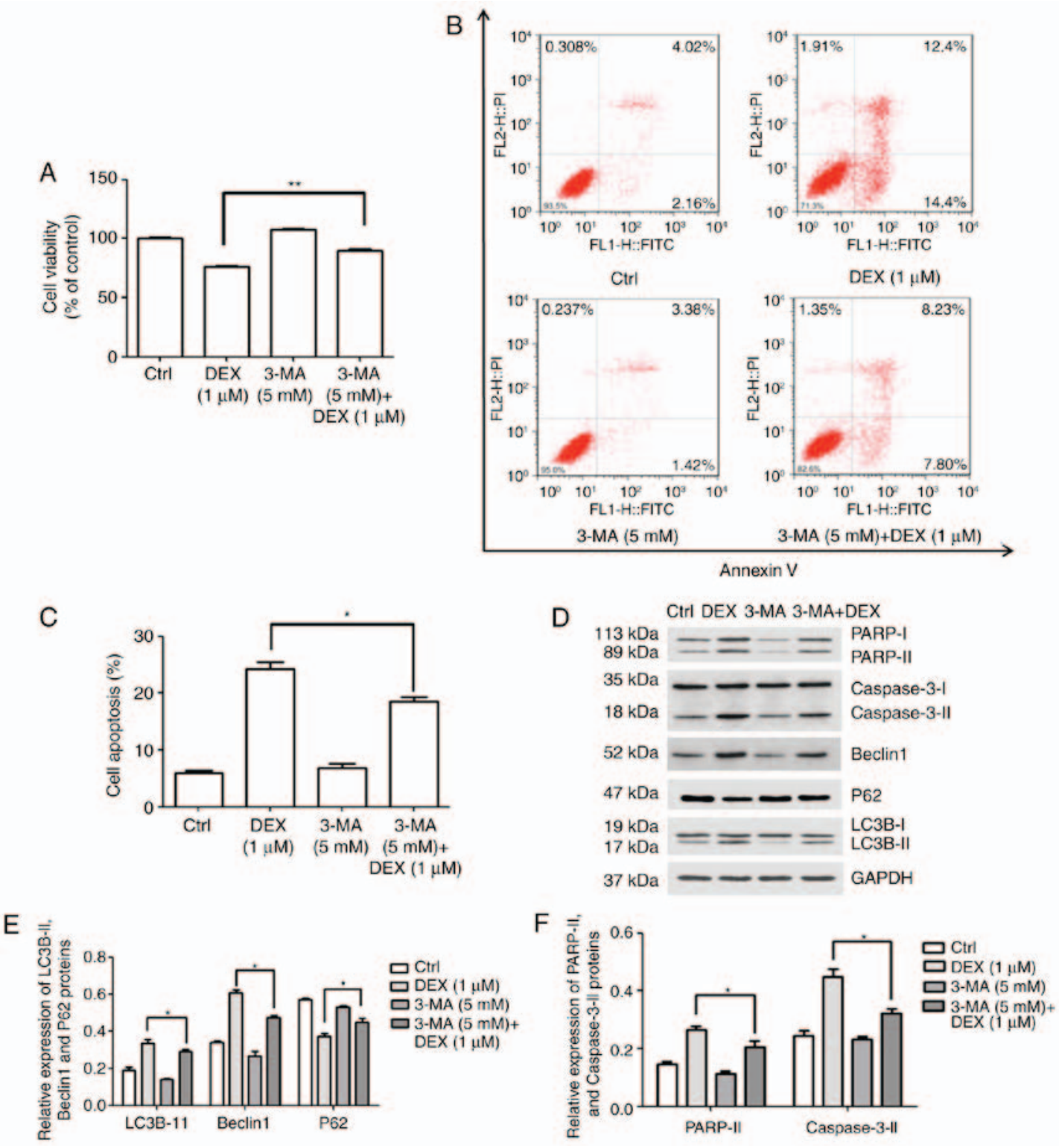

Figure 4. DEX promotes apoptosis by activating autophagy in MC3T3-E1 cells. (A-C) MC3T3-E1 cells were treated with DEX (1 $\mu$ M), 3-MA (5 mM) or 3-MA $(5 \mathrm{mM})+\operatorname{DEX}(1 \mu \mathrm{M})$ and $(\mathrm{A})$ the viability and $(\mathrm{B}$ and $\mathrm{C})$ the apoptotic rate were measured using a Cell Counting Kit-8 assay. (D) Western blot analysis of apoptosis-associated proteins (PARP and caspase-3) and autophagy-associated proteins (P62, LC3B and Beclin1). (E and F) The expression of each protein normalized to the GAPDH was evaluated. Values are expressed as the mean \pm standard deviation $(\mathrm{n}=3)$. " $\mathrm{P}<0.05$ and ${ }^{* *} \mathrm{P}<0.01$ vs. DEX ( $\left.\mu \mathrm{M}\right)$ group. Ctrl, control; ATF, activating transcription factor; DEX, dexamethasone; MA, 3-methyladenine; LC3B, microtubule-associated proteins 1A/1B light chain; PARP, poly[ADP-ribose] polymerase; FITC, fluorescein isothiocyanate; PI, propidium iodide.

ROS-associated stress. In addition, it was demonstrated that the expression of ATF4 and CHOP was also inhibited by NAC, indicating that ROS-associated stress also elevated ER stress $(\mathrm{P}<0.05$; Fig. 5H). Therefore, the effect of DEX on cell viability, ROS stress, apoptosis and protein expression was attenuated by NAC. These results demonstrated that DEX promoted the production of ROS, which enhanced apoptosis through the induction of autophagy and ER stress in MC3T3-E1 cells.

\section{Discussion}

GCs have excellent therapeutic effects and are among the most important drugs used in the clinic (18). However, GC therapy is often accompanied by severe side effects, including osteoporosis and osteonecrosis (19-21). As a side effect of their clinical applications, GCs cause bone mass loss, bone tissue degeneration and apoptosis of osteoblasts. The precise molecular mechanisms underlying DEX-induced apoptosis are yet to be fully elucidated. In order to investigate whether ER stress, ROS and autophagy are involved in this process, the production of ROS, ER stress and autophagy were investigated, and the effects of 3-MA and NAC on DEX-induced apoptosis in MC3T3-E1 osteoblast-like cells were assessed. It was demonstrated that autophagy promoted apoptosis, and that ROS induced ER stress and autophagy-mediated apoptosis. The present study provides a theoretical foundation for the mechanism of action of DEX, which comprises induction of apoptosis, ER stress, ROS and autophagy in osteoblasts, and may provide an alternative approach for molecular intervention 

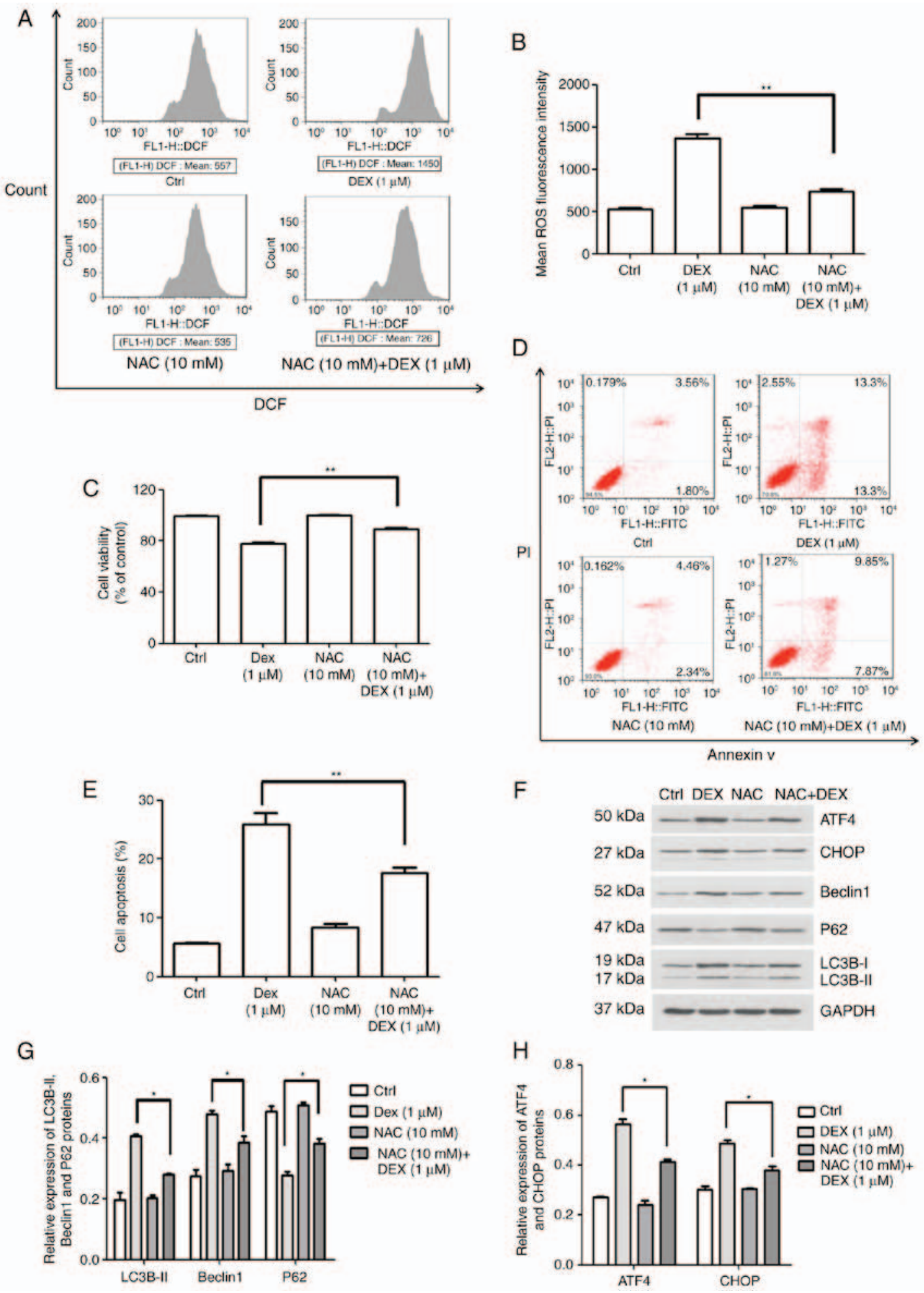

Figure 5. ROS production induced by DEX promotes apoptosis in MC3T3-E1 cells through ER stress and autophagy. (A and B) ROS production rate of MC3T3-E1 cells and quantification of it, in the control, DEX $(1 \mu \mathrm{M}), \mathrm{NAC}(10 \mathrm{mM})$ and NAC $(10 \mathrm{mM})+\mathrm{DEX}(1 \mu \mathrm{M})$ groups, as detected by flow cytometry. (C) Viability and (D and E) apoptosis after treatment with NAC. It was indicated that the activation of ROS was involved in the inhibition of viability induced by DEX. (F) Western blot analysis of autophagy-associated (LC3B, P62 and Beclin1) and ER stress-associated proteins (ATF4 and CHOP). (G and H) A relative quantification assay was performed to assess the expression of each protein normalized to the GAPDH expression Values are expressed as the mean \pm standard deviation (n=3). " $\mathrm{P}<0.05$ and ${ }^{* *} \mathrm{P}<0.01$ vs. DEX $(1 \mu \mathrm{M})$ group. Ctrl, control; ATF, activating transcription factor; CHOP, CCAAT/enhancer-binding protein homologous protein; DEX, dexamethasone; NAC, N-acetylcysteine; ROS, reactive oxygen species; ER, endoplasmic reticulum; DCF, dichlorofluorescin.

against DEX-induced apoptosis of MC3T3-E1 cells by inhibiting the production of ROS and autophagy.

In the present study, MC3T3-E1 cells were used to investigate the effect of DEX on osteoblast viability, apoptosis and cell cycle distribution. Previous studies suggested that DEX inhibited the viability of MC3T3-E1 cells in a dose-dependent manner, and that a concentration of $1 \mu \mathrm{M}$ DEX significantly decreased the viability of MC3T3-E1 cells (22-24). Additional 
studies revealed that $1 \mu \mathrm{M}$ DEX not only caused apoptosis, but also G0/G1 phase arrest $(22,23)$. These results demonstrated that DEX indeed inhibited the viability, and induced apoptosis and G0/G1 phase arrest in MC3T3-E1 cells. The present results are therefore consistent with those of previous studies. In addition, the expression of PARP and CDK 2 was detected in the present study. PARP is a downstream effector of the caspase family of proteins in the apoptotic pathway (25), and the cleavage of PARP after the activation of caspases has been identified as a biochemical hallmark of cellular apoptosis. Studies have demonstrated that DEX induces apoptosis and the expression of PARP in MC3T3-E1 cells simultaneously (26). CDK2 serves an important role in cell entry and transformation through $\mathrm{S}$ phase, as well as $\mathrm{G} 2$ phase transition to mitosis (27). The present results indicated that DEX may promote apoptosis by activating the cleavage of PARP and caspase-3, and that increased CDK2 expression may arrest the cell cycle of MC3T3-E1 cells at the G0/G1 phase.

In the present study, the impact of DEX on the production of ROS, ER stress and autophagy in MC3T3-E1 cells was investigated. The ER is an important organelle with a crucial role in a variety of cellular functions, including assembly, protein folding and $\mathrm{Ca}^{2+}$ storage. ER stress is a state in which the homeostasis of protein folding load and the capacity of the ER is disrupted $(28,29)$. Excess ER stress has been demonstrated in MC3T3-E1 cells after treatment with DEX, and has also been proven to induce apoptosis $(30,31)$. Autophagy is known to be a self-degradative process that is important for balancing sources of energy at critical times during development, and in response to nutrient stress (32). Studies have demonstrated that autophagy is involved in a number of general processes, including proliferation, development, apoptosis and cell death (33,34). LC3BII, Beclin1 and P62 are closely associated with the formation of autophagosomes, serving as markers of autophagy (35). Under normal physiological conditions, the cellular antioxidant system and the production of ROS are in a dynamic equilibrium, which has been demonstrated to serve a crucial role in the differentiation of osteoblasts (36). Disrupting this balance has certain harmful effects; for instance, an increased production of ROS may inhibit the differentiation of osteoblasts $(24,37)$. The results of the present study indicated that ER stress was activated by DEX via increasing the expression of the associated proteins ATF4 and CHOP, which is in accordance with the study by Yang et al (30). In addition, the results of the autophagy analysis indicated that DEX caused autophagy by increasing the levels of Beclin1 and LC3BII, and by decreasing the expression of P62. Furthermore, it was demonstrated that DEX increased the production of ROS in MC3T3-E1 cells, and this result is similar to the result described by Zhang et al (38).

In the present study, inhibition of autophagy by co-treatment with 3-MA significantly reduced the DEX-induced decreases in MC3T3-E1 cell viability. In addition, the protein activities of PARP and Caspase-3 were suppressed, indicating that DEX promoted apoptosis by activating autophagy. Of note, previous studies have revealed that autophagy is closely correlated with apoptosis, and that they interact in three ways, comprising cooperation, confrontation and promotion (39-41). Previous studies have indicated that relatively excessive accumulation of ROS serves a crucial role in the development and progression of apoptosis $(42,43)$, and under these conditions, cellular autophagy may be induced by transcriptional and post-transcriptional regulation (44). However, the association between autophagy and apoptosis induced by DEX in MC3T3-E1 cells has rarely been reported. The present study demonstrated that, in the experiment with the antioxidant NAC, the effects of DEX on viability and apoptosis were significantly suppressed, demonstrating that DEX promoted apoptosis by activating ROS signaling pathways in MC3T3-E1 cells. Furthermore, the expression of autophagy-associated proteins (Beclin1, LC3B-II and P62) and ER stress-associated proteins (ATF4 and CHOP) was detected, demonstrating that the effects of DEX on ER stress and autophagy in MC3T3-E1 cells were attenuated by NAC. These results indicated that DEX increased the production of ROS, which promoted apoptosis by activating autophagy and ER stress. Further study is required to confirm these results.

In conclusion, the present study demonstrated that DEX mediated ER stress, G0/G1 phase arrest, the production of ROS, autophagy and apoptosis in MC3T3-E1 osteoblast-like cells. Furthermore, production of ROS induced by DEX increased the level of autophagy, leading to MC3T3-E1 cellular apoptosis. Chloroquine, a lysosomal protease inhibitor, will be used in future studies to confirm the results of the present study. In addition, it is required to further elucidate the detailed mechanisms underlying the association between autophagy and apoptosis induced by DEX in MC3T3-E1 cells.

\section{Acknowledgements}

This study was supported by the Natural Science Foundation of China (grant nos. 81360273, 81460331 and 81560349).

\section{Competing interests}

The authors declare that they have no competing interests.

\section{References}

1. Kenanidis E, Potoupnis ME, Kakoulidis P, Leonidou A, Sakellariou GT, Sayegh FE and Tsiridis E: Management of glucocorticoid-induced osteoporosis: Clinical data in relation to disease demographics, bone mineral density and fracture risk. Expert Opin Drug Saf 14: 1035-1053, 2015.

2. Bultink IE, Baden M and Lems WF: Glucocorticoid-induced osteoporosis: An update on current pharmacotherapy and future directions. Exp Opinion Pharmacotherapy 14: 185-197, 2013.

3. Weinstein RS: Clinical practice. Glucocorticoid-induced bone disease. N Engl J Med 365: 62-70, 2011.

4. Weinstein RS: Glucocorticoid-induced osteoporosis and osteonecrosis. Endocrinol Metab Clin North Am 41: 595-611, 2012.

5. Kim J, Lee H, Kang KS, Chun KH and Hwang GS: Protective effect of Korean Red Ginseng against glucocorticoid-induced osteoporosis in vitro and in vivo. J Ginseng Res 39: 46-53, 2015.

6. Yun SI, Yoon HY, Jeong SY and Chung YS: Glucocorticoid induces apoptosis of osteoblast cells through the activation of glycogen synthase kinase 3beta. J Bone Miner Metab 27: $140-148,2009$.

7. Gu G, Hentunen TA, Nars M, Härkönen PL and Väänänen HK: Estrogen protects primary osteocytes against glucocorticoid-induced apoptosis. Apoptosis 10: 583-595, 2005.

8. Arai M, Shibata Y, Pugdee K, Abiko Y and Ogata Y: Effects of reactive oxygen species (ROS) on antioxidant system and osteoblastic differentiation in MC3T3-E1 cells. IUBMB Life 59: 27-33, 2007. 
9. Schröder K: NADPH oxidases in bone homeostasis and osteoporosis. Cell Mol Life Sci 72: 25-38, 2015.

10. Filaire E and Toumi H: Reactive oxygen species and exercise on bone metabolism: Friend or enemy? Joint Bone Spine 79: 341-346, 2012.

11. Wauquier F, Leotoing L, Coxam V, Guicheux J and Wittrant Y: Oxidative stress in bone remodelling and disease. Trends Mol Med 15: 468-477, 2009

12. Levine B and Kroemer G: Autophagy in the pathogenesis of disease. Cell 132: 27-42, 2008

13. Mizushima N, Levine B, Cuervo AM and Klionsky DJ: Autophagy fights disease through cellular self-digestion. Nature 451: 1069-1075, 2008.

14. Chu CC, Chua BH, Chen Z, Landy $\mathrm{C}$ and Hamdy RC: Dexamethasone induces caspase activation in murine osteoblastic MC3T3-E1 cells. Biochim Biophys Acta 1642: 79-85, 2003.

15. Biederbick A, Kern HF and Elsässer HP: Monodansylcadaverine (MDC) is a specific in vivo marker for autophagic vacuoles. Eur J Cell Biol 66: 3-14, 1995.

16. Eruslanov E and Kusmartsev S: Identification of ROS using oxidized DCFDA and flow-cytometry. Methods Mol Biol 594: 57-72, 2010.

17. Heckmann BL, Yang X, Zhang X and Liu J: The autophagic inhibitor 3-methyladenine potently stimulates PKA-dependent lipolysis in adipocytes. Br J Pharmacol 168: 163-171, 2013.

18. de Quervain D, Schwabe L and Roozendaal B: Stress, glucocorticoids and memory: Implications for treating fear-related disorders. Nat Rev Neurosci 18: 7-19, 2017.

19. Fowler TW, Acevedo C, Mazur CM, Hall-Glenn F, Fields AJ, Bale HA, Ritchie RO, Lotz JC, Vail TP and Alliston T: Glucocorticoid suppression of osteocyte perilacunar remodeling is associated with subchondral bone degeneration in osteonecrosis. Sci Rep 7: 44618, 2017.

20. Woolf AD: An update on glucocorticoid-induced osteoporosis. Curr Opin Rheumatol 19: 370-375, 2007.

21. Henneicke H, Gasparini SJ, Brennan-Speranza TC, Zhou H and Seibel MJ: Glucocorticoids and bone: Local effects and systemic implications. Trends Endocrinol Metab 25: 197-211, 2014.

22. Li H, Qian W, Weng X, Wu Z, Li H, Zhuang Q, Feng B and Bian Y: Glucocorticoid receptor and sequential P53 activation by dexamethasone mediates apoptosis and cell cycle arrest of osteoblastic MC3T3-E1 cells. PLoS One 7: e37030, 2012.

23. Lin H, Wei B, Li G, Zheng J, Sun J, Chu J, Zeng R and Niu Y: Sulforaphane reverses glucocorticoid-induced apoptosis in osteoblastic cells through regulation of the Nrf2 pathway. Drug Des Devel Ther 8: 973-982, 2014.

24. Lin H, Gao X, Chen G, Sun J, Chu J, Jing K, Li P, Zeng R and Wei B: Indole-3-carbinol as inhibitors of glucocorticoid-induced apoptosis in osteoblastic cells through blocking ROS-mediated Nrf2 pathway. Biochem Biophys Res Commun 460: 422-427, 2015.

25. Mcilwain DR, Berger T and Mak TW: Caspase functions in cell death and disease. Cold Spring Harb Perspect Biol 7: pii: a008656, 2015.

26. Li J, He C, Tong W, Zou Y, Li D, Zhang $\mathrm{C}$ and $\mathrm{Xu} \mathrm{W}$ : Tanshinone IIA blocks dexamethasone-induced apoptosis in osteoblasts through inhibiting Nox4-derived ROS production. Int J Clin Exp Pathol 8: 13695-13706, 2015.

27. Oakes V, Wang W, Harrington B, Lee WJ, Beamish H, Chia KM, Pinder A, Goto H, Inagaki M, Pavey S and Gabrielli B: Cyclin A/Cdk2 regulates Cdh1 and claspin during late S/G2 phase of the cell cycle. Cell Cycle 13: 3302-3311, 2014.
28. Iurlaro R and Muñoz-Pinedo C: Cell death induced by endoplasmic reticulum stress. FEBS J 283: 2640-2652, 2016.

29. Bánhegyi G, Baumeister P, Benedetti A, Dong D, Fu Y, Lee AS, Li J, Mao C, Margittai E, Ni M, et al: Endoplasmic reticulum stress. Ann NY Acad Sci 1113: 58-71, 2007.

30. Yang J, Wu Q, Lv J and Nie H: 4-Phenyl butyric acid prevents glucocorticoid-induced osteoblast apoptosis by attenuating endoplasmic reticulum stress. J Bone Miner Metab 35: 366-374, 2017.

31. Sato AY, Tu X, McAndrews KA, Plotkin LI and Bellido T: Prevention of glucocorticoid induced-apoptosis of osteoblasts and osteocytes by protecting against endoplasmic reticulum (ER) stress in vitro and in vivo in Female Mice. Bone 73: 60-68, 2015.

32. Glick D, Barth S and Macleod KF: Autophagy: Cellular and molecular mechanisms. J Pathol 221: 3-12, 2010.

33. Sui Y, Yao H, Li S, Jin L, Shi P, Li Z, Wang G, Lin S, Wu Y, $\mathrm{Li}$ Y, et al: Delicaflavone induces autophagic cell death in lung cancer via Akt/mTOR/p70S6K signaling pathway. J Mol Med 95: 311-322, 2017.

34. Yang M, Wang B, Miao L, Xu X and He X: Autophagy is involved in aldosterone-induced mesangial cell proliferation. Mol Med Rep 14: 4638-4642, 2016.

35. Pattingre S, Espert L, Biard-Piechaczyk $M$ and Codogno P. Regulation of macroautophagy by mTOR and Beclin 1 complexes. Biochimie 90: 313-323, 2008

36. Pawlowska E, Wysokiński D, Tokarz P, Piastowska-Ciesielska A, Szczepanska J and Blasiak J: Dexamethasone and 1,25-dihydroxyvitamin D3 reduce oxidative stress-related DNA damage in differentiating osteoblasts. Int J Mol Sci 15: 16649-16664, 2014.

37. Yang Y, Su Y, Wang D, Chen Y, Wu T, Li G, Sun X and Cui L: Tanshinol attenuates the deleterious effects of oxidative stress on osteoblastic differentiation via Wnt/FoxO3a signaling. Oxid Med Cell Longev 2013: 351895, 2013

38. Zhang S, Li D, Yang JY and Yan TB: Plumbagin protects against glucocorticoid-induced osteoporosis through Nrf-2 pathway. Cell Stress Chaperones 20: 621-629, 2015.

39. Ryter SW, Mizumura K and Choi AM: The impact of autophagy on cell death modalities. Int J Cell Biol 2014: 502676, 2014.

40. Mariño G, Niso-Santano M, Baehrecke EH and Kroemer G Self-consumption: The interplay of autophagy and apoptosis. Nat Rev Mol Cell Biol 15: 81-94, 2014.

41. Li M, Gao P and Zhang J: Crosstalk between autophagy and apoptosis: Potential and emerging therapeutic targets for cardiac diseases. Int J Mol Sci 17: 332, 2016.

42. Zhang Z, Zheng L, Zhao Z, Shi J, Wang X and Huang J: Grape seed proanthocyanidins inhibit $\mathrm{H}_{2} \mathrm{O}_{2}$-induced osteoblastic MC3T3-E1 cell apoptosis via ameliorating $\mathrm{H}_{2} \mathrm{O}_{2}$-induced mitochondrial dysfunction. J Toxicol Sci 39: 803-813, 2014.

43. Suh KS, Choi EM, Lee YS and Kim YS: Protective effect of albiflorin against oxidative-stress-mediated toxicity in osteoblast-like MC3T3-E1 cells. Fitoterapia 89: 33-41, 2013.

44. Li L, Tan J, Miao Y, Lei P and Zhang Q: ROS and Autophagy: Interactions and molecular regulatory mechanisms. Cell Mol Neurobiol 35: 615-621, 2015.

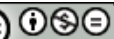

This work is licensed under a Creative Commons Attribution-NonCommercial-NoDerivatives 4.0 International (CC BY-NC-ND 4.0) License. 